\title{
PAUL VALÉRY: DE LA ESCRITURA A LA LECTURA
}

Carmen Camero Pérez

Universidad de Sevilla

\section{RESUMEN}

De la escritura a la lectura, el presente artículo propone un recorrido por la poética de Paul Valéry, a partir de sus escritos teóricos y de reflexión, sin olvidar los Cahiers y la Correspondance.

$\mathrm{El}$ análisis propuesto permite desvelar las líneas esenciales de un pensamiento, en el que la literatura encuentra su lugar y su justa medida, basadas en la desmitificación de un autor, cuyo prestigio se verá rechazado a favor de la singularidad y autenticidad, como marcas esenciales del texto.

Lejos del poeta oficial y académico, se dibuja el perfil de un Valéry teórico y crítico, cuyos principios de escritura producirán efectos análogos en la teoría de una lectura, que pone a los demás a prueba frente a su propio pensamiento.

Autor de un texto antirrealista y autorreflexivo, que sitúa en primer plano el proceso de la escritura, la autopoiesis valeriana nos permite asistir al análisis de un espíritu para el que el arte se constituye en medio privilegiado de conocimiento. Fundado en la dificultad, la paradoja, la duda, el profundo escepticismo y la enorme curiosidad de saber y conocer, Valéry nos ofrece a través de su obra la posibilidad de definir el sentido de su trayectoria y de su posible 
lectura, en un intento de conocer más y mejor ese rostro secreto del que sin duda fue un gran teórico de la literatura.

Il n'est pas sûr que l'entité $M$. Paul Valéry soit autre chose qu'une notation commode. 'Interrogarse sobre qué es el hombre y qué es el autor constituye una doble cuestión difícil de separar completamente, ya que nombramos «escritor» a aquél que escribe libros, pero mediante el término «autor» designamos un nombre y una obra, asociando pues la persona, el texto escrito y el escritor. Celui qui vient d'achever une oeuvre -escribe Valéry en Tel Quel-tend à se changer en celui capable de faire cette oeuvre. Il réagit à la vue de son oeuvre par la production en lui de l'auteur. Et cet auteur est fiction ${ }^{2}$. Crisis de la literatura que Valéry provoca deconstruyendo la imagen del autor, figura de la que nuestro autor no cesará de hablar, a partir de una experiencia de escritura ilustrada particularmente en los Cahiers. Es precisamente en estos Cahiers donde Valéry escribe: Le rapport d'une oeuvre à l'auteur est une des choses les plus curieuses. L'oeuvre ne permet jamais de remonter au vrai auteur. Mais à un auteur fictif ${ }^{3}$.

Reconstruido a posteriori, el autor alcanza el rango de mito, mito para él mismo y mito también para ese lector que no ve en el trabajo laborioso de la escritura que el fruto de la inspiración de las musas, reconstruyendo como causa del discurso a un autor capaz de emitirlo de manera espontánea, es decir un autor que, para Valéry, est infiniment peu probable. On appelait Muse cet auteur qui est dans l'auteur ${ }^{4}$.

Valéry desacraliza la noción de autor, amenazando su poder absoluto y alejándose de la posición dimiúrgica del autor inspirado del siglo XIX, contra la que habían reaccionado, a su manera, Baudelaire y Poe. El autor valeriano se sitúa en la práctica vivida en todos sus estados, debilidades y contingencias, llegando incluso a perder el reconocimiento y el poder de una paternidad que no parece estarle permitida, pues l'oeuvre dévore son père. Il n'en fut que le moyen. Elle le dépouille 5 . Más interesado por el procedimiento de la fabricación que por su resultado, Valéry hará desaparecer la imagen del autor "creador omnipotente», para afirmar la figura de un individuo en potencia, definido por su capacidad o voluntad de querer y de poder: J'ai agi toujours-escribe a su amigo Gide_- pour me rendre un individu potentiel C'est-à-dire que j'ai préféré une vie stratégique a une tactique. Avoir à ma disposition sans dispo-

\footnotetext{
1 Valéry, PaUl, (1960): Oeuvres II, Paris, Gallimard, «Pléiade», p. 1526

2 Ibid. p. 673

"Valéry, Pall ( 1974): Cahiers II. Paris, Gallimard, «Pléiade», p. 11

4 VALÉRY, PaUl ( 1960): Oeuvres II, ed. cit. p. 679

s VAlÉRY, PALl (1974): Cahiers II, ed. cit. p. 290
} 
ser. Ceci avait un but d'équilibre intime, imaginatif ${ }^{6}$. En efecto, muchos de los proyectos de Valéry, entre los que podemos citar su célebre Système son proyectos abortados, dada la dificultad valeriana de considerar algo acabado, dificultad que encontramos incluso en la conversación, hasta tal punto que, como señala Léautaud en su Journal Littéraire, Valéry era conocido como l'homme qui ne finit jamais.

Sobre la dificultad de Valéry para acabar un texto, la célebre anécdota contada por André Fontainas, según la cual el escritor habría ido a visitarle, pidiéndole ayuda para acabar el poema Eté, resulta bastante reveladora. Ya Maurice Blanchot, por otra parte, había señalado en 1949, cómo toute l'oeuvre de Valéry est entraînée vers une contradiction entre les termes de laquelle elle refuse de choisir, qu'elle répugne même à reconnaître pour une contradiction $^{\text {? }}$

Debatiéndose entre escribir o no escribir o más exactamente escribir sin escribir, Valéry muestra su desconfianza hacia la literatura, sin renunciar no obstante a la escritura. Nous ferions tant de poémes et de musiques et de tout sans noircir des feuillets ${ }^{8}$, confiesa a su amigo Pierre Louys en 1891, deseo del que también hará partícipe, tres años más tarde a Valery Larbaud: J'aurais bien voulu -le escribe-que nous fondassions une revue où il n'y aurait pas eu à écri$r e^{9}$. Consciente de la problemática que plantea el acto de escribir, Valéry llegará a convertirse en uno de los prosistas más brillantes de nuestro siglo. Una prosa que no es ni novelesca ni «narrativa», sino construida más bien en torno a los ensayos o las máximas. Considerando «escribir» como un problema, Valéry trabajará la forma, encontrando en tal actividad una verdadera excitación, que le lleva a amar su ejercicio: ce mot que j'ai mis sur La Jeune Parque -escribe a Jean Paulhan-est en réalité celui qui se met de lui même sur tout ce que je puis fai$r e^{10}$. Ejercicio que Valéry aplica para responder a los más diversos encargos, entre los que podemos citar su artículo de 1896 Une conquête méthodique, para el que, según palabras del mismo Valéry, nuestro escritor se veía privado tanto de los medios como del deseo de llevar a cabo algo tan alejado de sus propios gustos. El artículo será no obstante publicado, gracias a una operación de retórica patafísica, de la que Valéry hablará a Valette en una carta de 1915:

* Valéry, Paul ( 1955): Correspondance avec André Gide, Paris, Gallim

¿ $\mathrm{A}$ A

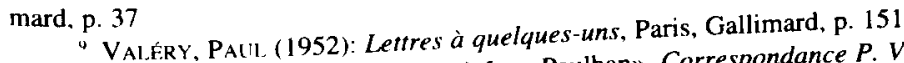

1" VAlF́RY, PAUL (1957): "Lettre à Jean Paulhan», Correspondance P. Valéry G. Fourment,

Paris, Gallimard, p. 244 
L'idée me vint enfin d'établir une correspondance entre l'organisation militaire allemande et l'économique. Personne n'y avait encore songé et j'étais sauvé! Ce rapprochement que je croyais in petto littéraire me donnait une base rhétorique (..) Mais l'histoire ne s'illumine et ne devient curieuse qu'en 1914. J'avais, en écrivant, la sensation que j'arrondissais, mécanisais et perfectionnais mes Boches. Et j'avais, peut-être, raison en ce temps là. Mais ils se sont révéles. Ils enfoncent même mon Boche idéal par des opérations de rhétorique pataphysique (..) Comme je ne connais ni l'Allemagne, ni l'allemand, ni quelque allemand, il en résulte qu'un point de vue tout arbitraire chez moi, et presque un acte de mauvaise conscience se change après coup en pressentiment de la vérité. Ceci est fort immoral.Et dire que je n'avais jamais beaucoup cru à la littérature".

Valéry, que se formó en la época de la segunda generación simbolista, en un medio impregnado de anarquismo y que contaba con gente como Pierre Louÿs, Alfred Jarry o Marcel Schwob, muestra en esta carta su faceta humorística, patafísica incluso, faceta poco conocida y bastante alejada del rostro del académico y del poeta oficial, que a menudo vemos en él. En este sentido cabe recordar que, como muy acertadamente señala Jean-Paul Goujon en un artículo de LÉtoile Absinthe ${ }^{12}$, les chroniques que Valéry donna pendant un temps à la revue d'Alfred Valette ne sont pas si éloignées qu'on pourrait le penser des spéculations de Jarry.

Volviendo a Une Conquête méthodique, señalemos que lo que realmente importa a Valéry no es la política del Estado, sino el principio racional que rige su industria, su ciencia, su comercio..., en una palabra, el método. Esta palabra clave en la poética valeriana aparece en repetidas ocasiones bajo la pluma del que llegaría a establecer una especie de vademécum para el escritor, que se enfrenta a las dificultades de la escritura en general y del trabajo por encargo en particular. Así, para ajustarse a la paginación del album en el que aparecería Eupalinos, Valéry se verá obligado a escribir un texto de 115800 caracteres, precisión que le llevaría a adoptar la forma del diálogo.

$\mathrm{Al}$ interés por tales exigencias cuantitativas, podemos añadir el placer que Valéry sentía cuando trabajaba su escritura, y que se pone de manifiesto en numerosos textos, entre los que cabe citar el siguiente de Ego Scriptor: Devant trop souvent écrire des choses dont je n'ai nulle envie et l'esprit inerte devant

" Carta citada por Thuillier, G. (1963): «Paul Valéry et la politique III», La Revue Administrative, mars-avril

12 Goujon, Jean-Paul (1984): «Un pataphysicien ignoré: Paul Valéry», L'étoile Absinthe 23-24, pp. $43-48$ 
elles, je m'avise de me donner les lettres initiales des phrases successives à faire comme pour un acrostiche ... Et ceci ferait scandale si je le disais ${ }^{13}$

Otorgando a la escritura este poder de transformación, Valéry anunciaría ciertos aspectos del Oulipo o del Nouveau Roman, por no señalar, los curiosos procedimientos de un Roussel, cuya obra era probablemente desconocida para Valéry. Poder de la escritura que explicaría la dificultad de Valéry para terminar una obra. Así parece deducirse del contenido de su lección 2 del Cours de Poétique, recientemente publicado en la revista Recherches Poḯtiques, con las fieles notas que Georges Le Breton había tomado para Yggdrassil: Toujours l'artiste connât l'envie de reprendre son oeuvre même publiée. Toujours le poème existe en son état. L'artiste abandonne l'oeuvre par dégoût et par ennui. L'esprit na jamais de quoi finir avec quelque chose ${ }^{14}$

Este carácter infinito del espíritu estaría en relación con la preocupación por el lenguaje, que obsesionó a Valéry durante toda su vida. En efecto, si en la lección 3 de este mismo Cours de Poétique, se afirma que el único medio de poner fin al caos del pensamiento es traducirlo en lenguaje, no por ello Valéry es menos consciente del hecho de que el origen desordenado y fortuito del lenguaje conlleva no pocas imprecisiones y no responde a la naturaleza de las cosas. Una reflexión de este tipo entraña diversas consecuencias que Richard Conte, siguiendo a Judith Robinson, se ha encargado de señalar ${ }^{15}$ En primer lugar, las palabras interponen una barrera artificial entre el espíritu y las cosas, haciéndonos ignorar todo aquello que carece de nombre. Pero la razón principal por la que Valéry desconfía de las palabras, es justamente porque crean cuestiones artificiales, problemas ilegítimos puramente linguísticos. A nivel de la poiética, como filosofia del acto creador, habría pues que inventar, o mejor reinventar un lenguaje nuevo, que pudiera dar cuenta del análisis del espíritu en acto. Ahora bien, el lenguaje introduce igualmente una problemática temporal en la escritura, que corre tras las sombras de la conciencia, dado que siempre se escribe en pasado, con lo que la actualidad del yo nunca es alcanzada, sino más bien disimulada por la escritura.

Tal idea de la práctica de escritura explica la existencia en Valéry de una poesía virtuosa y trabajada, que se arma de técnicas y límites formales. Esfuerzo sobre la forma de un escritor que, siguiendo la lección de Baudelaire y de Poe, se complace en ofrecemos la imagen de un poeta crítico.

En su reflexión teórica sobre la génesis del acto creador, Valéry distingue dos momentos, el de la invención y el de la elección crítica. Poésie et pensée

1.3 Valéry, Paul (1973): Cahiers I, Paris, Gallimard, «Pléiade», p. 273

14 VAléry, PAUL (1996-97): «Leçon 2 du Cours de Poétique», pronunciada el 11 de diciem-

bre de 1937. Recherches Poïétiques 5, p. 7

is CONTE, RichaRd (1996-97): «La poétique de Paul Valéry», Recherches Poḯtiques 5, p. 43 
abstraite, la Première Leçon du Cours de Poétique o Les Propos sur la poésie tienden a restar importancia al primer momento, en el que un ser recibe el don de las musas y del azar, para actuar como un vulgar «medium». Al poeta profeta, Valéry preferirá el poeta crítico, consciente y responsable de su acto de creación, actitud que ha caracterizado a Valéry desde sus primeras composiciones poéticas: Jamais —escribe a su amigo Louys en 1890-mon idéal artistique ne s'abandonnera aux hasards de l'inspiration, jamais il n'écrira tout un poème dans une nuit de fièvre ${ }^{16}$.

Su desconfianza con respecto a la facilidad de la improvisación aparece a menudo en sus cartas a André Gide a partir de 1891. Así, a propósito de Narcisse parle, Valéry escribe:

J'ai pris ma plume et me voilá dans les affres. Car le Narcisse longuement rêvé ne devrait se faire que minutieusement à courtes heures! Et je souffre de le sentir s'augmenter facilement presque (...) Car cela ne pourra rien valoir si hâtivement fait. Mais vous ne vous imaginez pas quel déchirement ${ }^{17}$

No sería pues fruto del azar, el hecho de que a propósito de sus Fragments de Narcisse, Valéry declare igualmente a su amigo Gide:

(Ces huit derniers vers) que vous citez là, sont très précisément ceux qui $m$ 'ont coûté le plus de travail et que je considère comme les plus parfaits de tous ceux que j'ái écrits, je veux dire les plus conformes à ce que j'avais voulu qu'ils - fussent, assouplis à toutes les contraintes que je leur avais assignées ${ }^{18}$

De tales consideraciones podemos deducir que la mejor poesía no es precisamente la que proviene del genio de la inspiración, sino más bien del trabajo consciente e inteligente. La fascinación del arte no reside en la espontaneidad del genio, sino en la conciencia del trabajo: Je n'aime que le travail du travail (..) Le spontané, même excellent ne me semble jamais assez mien, ${ }^{19}$ escribe Valéry a propósito del Cimetiére marin, oponiéndose totalmente al Surrealismo. Más que el genio, a Valéry le seduce el trabajo para llegar a alcanzar un arte propio, o más exactamente para alcanzar la conciencia de sí mismo.

is Citado por Hytier. Jean (1953): La Poétique de Valéry. Paris, A. Colin, p. 133

17 VALÉRY, PAUL (1955): Correspondance avec André Gide, op. cit. pp. $48-49$

18 Citado por Latour, J. DE (1935): Examen de Valéry, précédé d'une lettre et d'un texte inédit de $P$. Valéry, Paris, Gallimard, p. 159

14 Valéry, Paul (1957): "Au sujet du Cimetière marin», Oeuvres I, ed. cit. p. 1500 
Ya en 1919, este interés se afirmaba de forma bastante clara en su ensayo sobre Leonardo: (dans l'art) je ne mettais rien au-dessus de la conscience, -j'aurais donné bien des chefs-d'oeuvre que je croyais irréfléchis pour une page visiblement gouvernée ${ }^{20}$.

Primacía pues de la conciencia y la reflexión en la producción de un escritor que distinguirá los versos encontrados de los elaborados, considerando que si bien hay que perfeccionar los primeros, también es preciso naturalizar los segundos. Doble simulación en sentido inverso, dado que el valor del poeta reside en su trabajo consciente, que comprende la capacidad de saber elegir las sugestiones de la inspiración, eliminando la impresión de esfuerzo que la obra pudiera sugerir.

En cierto sentido, como bien señala Luigi Pareyson ${ }^{21}$, la inspiración concierne más al lector que al autor, ya que no se trata de una cualidad del autor, sino de una idea que debe suscitar en el lector como explicación de esa facilidad que se desprende de su obra. De esta forma, Valéry no llegaría a negar totalmente la doctrina de la inspiración, a la que hace, como vemos, ciertas concesiones. Por otra parte, el privilegio que se otorga al trabajo de la forma, no impide conferir a ésta la sensación de presencia de un cuerpo y una voz. Valéry defendería así una concepción sensible de la obra, cercana a la que Roland Barthes ${ }^{22}$ expone en su Degré zéro de l'écriture, donde el estilo se define como una hypophysique de la parole donde el cuerpo aflora bajo la forma al igual que la sangre bajo la piel. Curioso paralelismo, señalado por Michel Jarrety ${ }^{23}$, entre el autor del Degré zéro y el del Cimetière marin, que no dudó en poner en la petición de beca hecha por Barthes para el liceo Louis Le Grand, la apostilla siguiente: Je recommande très chaudemente cette candidature à $M$. Le Ministre. Le 29 novembre 1933. Paul Valéry.

Cincuenta años más tarde será Barthes quien hablará de Valéry, colaborando en el dossier que Le Magazine Littéraire dedicó al autor de Charmes. Le probléme posé par la faqon dont Valéry écrit -señala Barthes--est, qu'en réalité, son écriture est masquée par quelque chose qui, maintenant, n'est plus pensée et qui est le style. Il écrit avec du style ou le style ${ }^{24}$.

Sobre la problemática del estilo, Valéry se había pronunciado ya a la edad de dieciocho años en su artículo sobre la técnica literaria: Le style n'est pas un

20) Val.éry, Paul (1957): «Variété», Oeuvres I, ed, cit, p. 1206

(1996-97): «Deux essais sur la poétique de Valéry»,Recherches Poüétiques 5. op. cit. p. 6

22 Barthes, Roland (1959): Le degré zéro de l'écriture. Paris, Le Seuil, p. 19

23. JARRETY, MiChel (1991): Valéry devant la littérature. Paris, P.U.F. p. 171

24 BARTHES, Roland (1978): "Valéry vu par Roland Barthes», propos recueillis par M. Benézet et J.-L. Schefer. Repris dans Magazine Littéraire, octobre, 1982, pp. 29-31 
rite inavariable, un éternel moule définitivement coulé (..), il doit se plier au dessein de l'auteur et servir uniquement à préparer le feu d'artifice final Il le faut adéquat a l'objet ${ }^{25}$. Perspectiva de artificiero, sin duda marcada por la herencia de Poe y que se centra más en el trabajo del objeto que en la naturaleza del sujeto escritor. Sin embargo, tal posición se verá invertida hacia 1920 , fecha a partir de la cual el estilo pasará a significar la manera de la que uno se expresa, manera que es significativa de su singular naturaleza. Un doble objetivo se instaura así para mantener el efecto y la singularidad, dando lugar a lo que Valéry llama « las dos caras del estilo»: approcher de soi et agir sur l'autre ${ }^{26}$, mediante la relación que el autor establece con su propio uso del lenguaje. Ahora bien, este lenguaje es al mismo tiempo su medio y su obstáculo, y el estilo se basa en este conflicto mantenido en el interior del mismo lenguaje, en el que se trabaja con vistas a hacer que lo común se convierta en singular. En efecto una de las mayores preocupaciones de Valéry era encontrar una palabra que fuese verdaderamente suya, a pesar de que él mismo sabía que en la palabra del poeta siempre hay quelque livre mort qui s'agite et reparle ${ }^{27}$. Encontramos así planteada la cuestión de la originalidad, problema antiguo, pero siempre actual, sobre el que Valéry se confiesa en su Lettre sur Mallarmé:

Nous disons qu'un auteur est original quand nous sommes dans l'ignorance des transformations cachées qui changèrent les autres en lui; nous voulons dire que la dépendance de ce qu'il fait à l'égard de ce qui fut fait est excessivement complexe et irrégulière ${ }^{2 x}$.

Confesión sincera de un Valéry que hará de la cuestión de la herencia una de sus preocupaciones. Así, hablar de sí mismo en tanto que autor, consistiría en decir en qué medida me han formado los demás y cómo he adaptado su legado. A este respecto, podemos recordar la reflexión que hace Valéry en Situation de Baudelaire:

L'homme ne peut qu'il ne s'approprie ce qui lui semble si exactement fait pour lui qu'il le regarde malgré soi comme fait par lui ... Il tend irrésistiblement à s'emparer de ce qui convient étroitement à sa personne; t te langage même confond sous le nom de bien la notion de ce qui est adapté á quelqu'un et la satisfait entièrement avec celle de la propriété de ce quelqu'un ${ }^{29}$.

2s VALERY, PAUL (1957): "Sur la technique littéraire», Oeuvres I, ed. cit. p. 1830

26 VALERY, PAUL (1973): Cahiers I, ed. cit. p. 311

27 Valéry, Paul (1960): «Littérature», Oeuvres II, ed. cit. p. 562

28 VAlÉRY, Paul (1957): «Lettre sur Mallarmé», Oeuvres I, ed. cit. pp. 634-635

29 Valéry, Paul (1957): «Situation de Baudelaire», Oeuvres l, ed. cit. pp. 607-608 
En esta defensa de un Baudelaire heredero de Poe, Valéry intenta, quizás defenderse a sí mismo. Sus fórmulas, frases o ideas, que a veces toma prestadas a sus predecesores ${ }^{30}$, muestran a un Valéry fiel a ese pensamiento que en 1916 había escrito en su Cuademo: Rien de plus original: Rien de plus soi que se nourrir des autres. Mais il les faut digérer. Le lion est fait de mouton assimilé $^{31}$. Metáfora que permite al poeta negar una originalidad en la que no cree en absoluto, ya que como él mismo dirá: Il n'y a pas d'écrivains originaux, car ceux qui mériteraient ce nom sont inconnus; et même inconnaissables $^{32}$. Se muestra así de nuevo la mirada constante de un Valéry que se dirige más a la fabricación que a su resultado, mostrando los diversos aspectos del falso semblante, ya que en efecto aquellos que quieren preservar su originalidad sólo obedecen a quienes le han hecho creer en el valor de la misma. Principio extraño para un poeta como Valéry, cuya ética consiste más bien en intentar escribir enfrentándose al deseo de novedad y de seducción, dado que lo que verdaderamente le preocupa es tomar partido por encontrarse a sí mismo, objetivo que pondrá en práctica mucho más en sus Cuadernos que en sus Obras.

En 1892, después de haber publicado su Narcisse parle en la Conque, Paul Valéry se retira de la poesía, a la que volverá veinte años más tarde, ofreciendo a su amigo Gide La Jeune Parque, a la que seguirán los magníficos Charmes. Sin embargo, durante todo este tiempo, Valéry seguirá construyendo su obra, mediante toda esa monumental masa de notas y reflexiones que constituirán sus Cahiers. Comenzados a partir de 1894, estos Cuademos constituirán la matríz secreta de todas las obras que posteriormente publicará, configurando ese espacio íntimo, preferible al de sus obras públicas, más ligadas al espíritu mundano. En el espacio privilegiado de los Cahiers, Valéry encuentra el medio favorable para el ejercicio de su pensamiento, encontrándose mucho más cerca del acto de creación, al ofrecer una transcripción más viva de su pensamiento, más libre y espontáneo. Nada más alejado, en efecto, del trabajo previsto y encargado, sometido siempre al juicio de otro. El espacio secreto de los Cahiers hará surgir la imagen de un autor sin lector y sin obra, que se distancia del yo contingente, revelando un yo distinto al mundano y público, que a menudo asignamos a Valéry, personaje que él mismo definirá como ser emnascarado y ficticio.

Tu l'imagines, chaque matin, il se lève a l'aube, prépare seul sa ration de café, chaque matin il se déclare son plaisir à cela et au tabac

30 Cf. Hytier, JeAn (1967): Questions de littérature. Genève, Droz, pp. 141-158

3 Valéry, Paul (1974): Cahiers II, ed. cit. p. 1003

12 Valéry, Paul (1960): Oeuvres II, ed. cit. p. 677 
qu'il fume, seul, face à la page blanche; chaque jour une même obsession -de quoi?, de qui? Pourquoi cette reprise indéfinie? ${ }^{3.3}$

Tales cuestiones encuentran rápida respuesta si recordamos el rechazo de la literatura del que Valéry hará partícipe a su amigo Gide:

J'en reviens toujours á dire: la littérature ne peut avoir que trois fins. C'est-à-dire que quoi qu'on en fasse, elle aboutit aux trois classes suivantes(..) $1 .^{\circ}$ galette, $2 .^{\circ}$ nombre d'individus qui savent votre nom, 3. instruction personnelle, par les problémes d'ordre général auxquels conduisent la technique et l'exercice de l'art.

let 3 ont mes sympathies. Tout préférable á 2 (sauf en ce que 2 donne (). Si l'on adopte 3 , ou bien on quitte la littérature ou bien on la garde, mais à titre particulier, épisodique ${ }^{34}$

Despreciando al número de individuos que conocen su nombre, Valéry renuncia a una gloria, que sin embargo le ha sido asignada. Rechazo pues de una carrera literaria vana, que permitiría separar sus Obras de sus Cuademos. Si las primeras muestran el signo de ese profundo tedio que Valéry confiesa a menudo a su amigo Gide, los segundos aparecerán siempre asociados al placer que procura la búsqueda del conocimiento personal. Je suis beaucoup plus satisfait de mes éternelles notules que d'Agathe —escribe Valéry a Gide en $1901{ }^{35}$; su Narcisse es considerado déplorable, Celle qui sort de l'ombre es un soneto moins que quelconque, mientras que con Teste en Chine, on s'ennuie ensemble ${ }^{36}$.

Se diría que Valéry, después de haber renunciado a la literatura, siente el dolor y la vergüenza de escribir a pesar de todo. Así, como bien sugiere Gérard Genette ${ }^{37}$ toda la obra de Valéry no sería en definitiva más que un imnenso post-scriptum, que bien puede recordar los casos de un Francis Ponge o un Maurice Blanchot. El rechazo de 1892 parece constituir un deseo de transparencia consigo mismo, que explicaría, por otra parte, la adopción de esa escritura íntima, que renuncia a la transmisión y a la publicación: Je n'aime pas les idées des autres, et c'est pour ne pas faire des miennes les idées des autres que je ne les ai pas publiées ${ }^{38}$.

En definitiva, el problema, como Vincent Kaufrnann señala muy acertadamente ${ }^{39}$, no reside en la publicación, sino más bien en la autoría y el valor

33 BénÉzet, Mathieu (1982): «Paul Valéry moi-même», Magazine Littéraire, n. ${ }^{\circ}$ cit. p. 28

${ }^{34}$ VALÉRY, PAUL (1955): Correspondance avec André Gide. Op. cit. p. 364

35 Valéry, Paul, ibid. p. 386

s6 Ibid. pp. 50, 103 y 350 respectivamente

"Genette, Gerard (1976 ): «La littérature comme telle», Figures 1. Paris, Le Seuil, p. 263

3* Valéry, Paul (1973): Cahiers I, ed. cit. p. 258

39 KaUfmann, VinCEnT (1986): Le livre et ses adresses, Paris, Klincksieck, pp. 151-176 
contractual. Siendo algo inmediato, la enunciación en los Cahiers no tiene que ser ni representada, ni legitimada. Se trata de una escritura que no pasa contrato, dado que se encuentra ligada al mismo sujeto. Si Valéry se dirige al otro, lo hace de manera cínica, para que sea el lector quien asuma las responsabilidades del texto.

En sus Cahiers Valéry plantea el problema de la identidad, aunque no se trata en ningún caso de un sujeto biográfico, sino de un coeficiente de existencia absolutamente abstracto. De ahí que la imagen que podamos deducir sea la de un Valéry revisado y corregido por Valéry, es decir, transformado en un personaje de Valéry. Toma de conciencia y autoconocimiento, que Valéry practica para escapar a las formas establecidas, sin renunciar por ello a la inserción social (Academia, conferencias, prólogos, etc.).

Tal actitud permitiría explicar la postura de Valéry frente a un lector, que puede amenazar al autor, introduciendo en su yo la idea de alteridad. Asumiendo el papel del lector anticipadamente, el autor integra el efecto de lectura en la escritura, haciendo que su obra actúe sobre sí mismo, lo que explicaría el inquietante fenómeno del creador creado. De ahí que el consumidor, como Valéry lo había formulado ya en la Primera Lección de su Cours de Poétique, se convierta a su vez en productor: producteur d'abord de la valcur de l'ouvrage, et ensuite (..) producteur de la valeur de l'être imaginaire qui a fait ce qu'il admire 40 .

Al concebir la lectura como un acto de asimilación, consistente en asumir el papel del que habla, se comprende con facilidad que para Valéry en todo lector ideal se encarne una parte del escritor. Tal lector será en parte aquél que el escritor hubiera querido ser, lo que nos permite reconstruirlo, o más bien, elaborar el autorretrato deValéry lector, a partir de sus propios fragmentos y reflexiones teóricas.

Recordemos que después de la publicación de Charmes, en 1922, más que el Valéry poeta, será el Valéry crítico, autor de comentarios y prólogos, quien se muestre ante el público. Pero no insistiremos aquí sobre estos trabajos de subsistencia y encargo a los que ya nos hemos referido antes, con el fin de penetrar mejor ese otro aspecto, más interesante y menos conocido, relativo a las relaciones de Valéry con la crítica, así como al retrato de Valéry como «lector» de otros.

En 1928, el encuestador Maurice Rouzaud había planteado a diversos escritores la siguiente pregunta: Où va la critique?, pregunta a la que Valéry respondio sin dudar un instante: $A$ sa perte, j'espère. Opinión francamente desfavorable acerca de un trabajo que a pesar de asegurarle la vida y el 
reconocimiento, considera inútil, imposible e incluso dañino. Considerando que la crítica jamás puede llegar al secreto de la creación, que la biografía es más fácil que el análisis y que es prácticamente imposible alcanzar el verdadero ser, Valéry atacará toda crítica ignorante de su apariencia y su falsedad. El prólogo que él mismo escribe para el comentario que Gustave Cohen realizara sobre el Cimetière marin resulta a este respecto bastante revelador: Mon pò̀me étudié comme un fait accompli, révélant á l'examen de l'expert sa composition, ses intentions ses moyens d'action, sa situatuion dans le système de l'histoire littéraire, ses attaches et l'état probable de l'esprit de son auteur. Prólogo como vemos bastante adulador para el crítico en cuestión, que no renuncia sin embargo a mostrar, mediante el empleo del término «probable», la imposibilidad de captar el verdadero ser del poeta. El estado probable del espíritu de su autor no es en definitiva más que la apariencia, el parecer de una obra y de un creador difícil de desenmascarar.

Valéry alaba no obstante toda crítica capaz de captar el trabajo, el ejercicio del autor y el problema que se ha planteado, transponiendo así a la crítica sus ideas de creador, es decir que en la lectura de una obra, Valéry se interesará por descubrir el sistema que pudo crearla. Esto es precisamente lo que intentó hacer en su Introduction à la méthode de Léonard, intentando moldear su propio retrato. Une oeuvre qui m'intéresse profondément est une oeuvre qui m'excite à me figurer le système vivant et pensant qui l'a produite ${ }^{41}$. El autor se convierte así en pura ficción, imaginación de un sistema que Valéry descubre, haciendo uso de él, lo que explica el interés por el pastiche y la lectura apropiación. Se comprende por tanto que Valéry sólo admire los libros que él mismo hubiera sido incapaz de escribir, terminando por no soportar más que les livres qui sont les plus contraires à (son) tempérament, précisément parce $q u$ 'ils vous en tirent et qu'on est excédé de soi ${ }^{42}$.

$\mathrm{Ni}$ erudito ni historiador, sino artista y escritor, Valéry sólo juzga a partir de sí mismo, a pesar de la relatividad que tal punto de vista puede conllevar, practicando una crítica parcial, personal e incluso bastante osada. Alejándose de la crítica profesional sometida a los caprichos de la moda y de la novedad, Valéry no duda en establecer sus preferencias y confesar sus opiniones, incluso si corre el riesgo de chocar a su lector. Así si examinamos la lista de escritores que le interesaron (André Lebey, Lucien Fabre, Mathilde Pomès, André Caselli, etc.), sorprende comprobar que, a excepción de André Breton, Valéry no apoyó a ningún escritor importante. Se diría incluso que estaba «ciego» frente al talento de algunos de sus contemporáneos, cuya reputación estaba ya

41 Valéry, Paul (1960): Oeuvres II, ed. cit. p. 1539

42 VALÉRY, PAUL (9 avril 1914): Lettre inédite à Pierre Louÿs, colección particular 
bastante consolidada. Tal fue el caso, por ejemplo del homenaje a André Gide, que Valéry rechazó, alegando sus enormes y fatigosas ocupaciones. Es posible que la amistad jugase a favor del autor del Journal, que Valéry consideraba realmente como una nulidad. Sin embargo, el autor de La Recherche no correría idéntica suerte. L'Hommage à Proust que Valéry improvisa en Neuchâtel en 1922, muestra un desconocimiento casi total de la obra de su contemporáneo, que Valéry confiesa por otra parte, y tranquilamente, no haber leído verdaderamente. En efecto, cuando en 1940, nuestro autor entra de lleno en La Recherche, durante una semana de gripe, su opinión no difiere mucho de la que ya poseía antes: Quel délayage! Que cest déglingué! Et du Goncourt un peu partout. Avec ça, trop de pédérastie, musquée ou masquée, comme ils voudront,pour une substance assez maigre et même, avec ses amplifications magnifiques, un peu passée 4 .

Rechazo total de Proust en un homenaje a Proust, que muestra bien que Paul Valéry no temía en absoluto confesar sus gustos particulares, aún a riesgo de situarse abiertamente a contra corriente. Por otra parte, estos comentarios resultan altamente chocantes, si tenemos en cuenta que Valéry no leía verdaderamente, sino que más bien hojeaba los libros, convencido de haber captado su funcionamiento al cabo de algunas páginas, abandonando pues rápidamente todo lo que a sus ojos no iba a aportarle nada interesante. Tal parece haber sido el caso de Montaigne, si tenemos en cuenta el comentario que el mismo Valéry hace en sus Cahiers: (...) j'ai ouvert un Montaigne: En peu de minutes je l'ai renvoyé. Il m'assommait. Tout le monde peut écrire de ces choses ${ }^{44}$.

Del mismo modo que señala sus dudas o repugnanacias, Valéry se encargará también de hacernos partícipes de sus preferencias. En este sentido, más que por el Gran Siglo de Racine o de La Fontaine, Valéry siente predilección por el siglo XVIII, con su razón crítica y su desenvoltura, como bien lo muestran los textos de Variété dedicados a Montesquieu y a Voltaire. No obstante, su más claro amor se dirige a Stendhal, al que Valéry alaba en un extenso prólogo a Lucien Leuwen. Después de haber leido con gusto, durante su juventud, la Vie d'Henry Brulard, les Souvenirs d'égotisme y Lucien Leuwen, Valéry alaba el hecho de que Stendhal haya practicado l'acroissement de la conscience de soi, l'observation constante de soi-même ${ }^{45}$. En el fondo, Stendhal había hecho lo que Valéry, por su parte, también realizará, al cultivar su propia excepción, de ahí que Valéry vea más allá del escritor y del novelista. Para él, lo más sorprendente en Stendhal es el tono que se revela como el más individual de

4.3 Citado por Mondor, Henri (1957): Propos familiers de Paul Valéry, Paris, Grasset, p. 228

4 Valéry, Paul (1973): Cahiers I, ed. cit. p. 206

45 VAlÉRY, Paul (1957): Oeuvres I, ed. cit. p. 558 
toda la litreratura: Henry Beyle est à mes yeux un type d'esprit bien plus qu'un homme de lettres. Il est trop particulièrement soi pour être réductible à un écrivain. C'est en quoi il plaît et déplaît et me plaît ${ }^{46}$.

Buscando ante todo calidad y perfección, término éste que ya había sido empleado a propósito de Mallarmé, Valéry repartirá sus admiraciones entre Baudelaire, Mallarmé, Hugo, Huysmans, Voltaire, Nerval, sin que sus gustos sean, por otra parte inmutables. Citaremos, a título de ejemplo, dos casos reveladores de desplazamiento doble del sujeto y del objeto, que nos remiten a las teorías modemas sobre la verdad, desde la hermeneútica de Gadamer y el indeterminismo de Popper. El primer caso, que tomamos prestado de la obra misma de Valéry, se refiere a Stendhal: Je viens de relire Lucien Leuwen, qui n'est pas tout à fait celui que j'ai tant aimé il y a 30 ans. J'ai changé et il a changé $e^{47}$.

Para el segundo caso, citaremos una sabrosa carta inédita de Valéry a Pierre Louys, del 11 de septiembre de 1914, en la que se amortigua el entusiasmo que el poeta había confesado a su amigo, sobre el mismo escritor, siete meses antes:

(..) Quant à Victor Hugo, veux-tu mon sentiment? -Je le prends à ma guise-je le décime et me le filtre. Vraiment je n'oserai te représenter un Hugo selected by me. Tu me traiterais à la Boche, étant infiniment dangereux quand tu tiens ton Hugo à la main: tu en extrais des millions d'irréfutables beautés; et quand on y retourne tout seul, on ne les retrouve jamais.

Je te soupçonne d'avoir fabriqué tout ce qu'il y a de bien dans Hugo. Tout au moins, tu y en remets!.

Ceci dit,je prétends que j'admire en lui l'étonnant «réaliste»qu'il est.

Cet homme passe pour un lyrique! Et le lyrisme est celui de ses faibles qu'il croyait son fort. Erreur fréquente et grave.

Quand je pressens ses tonnerres, ses Jéhovah, ses infinis à douze dimensions, ses soleils noirs ... et quand je vois venir les apostrophes, l'anathème, les définitions toquées, les dégueulades trop longues, trop riches, trop ironiques, trop bonnes, trop violentes, ce trop me fait rire.

Pourquoi? -Mon "sentiment critique» prend souvent la forme familière suivante: -Toi, mon vieux (Hugo ou autre) - tu n'as pas réellement pensé tout ça. Tu es un de mes spéculateurs sur les mots, comme il en est qui spéculent sur le coton, dont ils n'ont jamais vu l'ombre d'une balle. Spéculateuur, tu as sans doute un estomac pro-

4h Ibid. p. 582

47 lbid. p. 535 
digieux, -mais si enfin je te somme de livrer ou de payer, -il n 'y a plus grand monde ... Mais je ne te reproche de spéculer, -entendonsnous!-je te reproche de te laisser pincer ${ }^{48}$.

De la escritura a la lectura, este breve recorrido por la poética de Valéry permitirá subrayar, a modo de conclusión, las líneas esenciales de un pensamiento, en el que la literatura encuentra su lugar y su justa medida. La desacralización de la autoridad se ve reforzada por la desacralización del autor, dado que el análisis de Valéry rechaza la idea de un prestigio que sólo se funda en la tradición de una confianza. El escritor y teórico preferirá sustituic ésta por una autenticidad a fin de que en toda obra pueda descifrarse el poder original de ese alguien con quien el lector entra en litigio.

Más allá de toda traducción posible en un lenguaje compartido, es el texto el que lleva siempre la marca de la singularidad. Principios de escritura que producen efectos análogos en la teoría de la lectura, dado que leer consiste en resistir al poder del autor, apropiándose de lo útil y despreciando lo inútil, ya que se trata de poner a los demás a prueba frente a su propio pensamiento. Es lo que, en mi opinión, Paul Valéry, llevado a «parecer» y empujado a «ser» no cesó de practicar durante toda su vida.

${ }^{4 x}$ Lettre inédite de Paul Valéry à Pierre Louÿs. Collection particulière 